\title{
Comparison of Patient Perceptions with Diode Laser and Scalpel Technique for Frenectomy
}

\author{
Dr. Soni Bista, ${ }^{1}$ Dr. Khushbu Adhikari, ${ }^{1}$ Dr. Charanjeet Singh Saimbi, ${ }^{1}$ Dr. Bikash Agrahari ${ }^{1}$ \\ ${ }^{1}$ Department of Periodontology and Oral Implantology, Universal College of Medical Sciences, College of Dental Surgery,
}

Bhairahawa, Nepal.

\begin{abstract}
Background: Frenum that encroaches on the margin of the gingiva may interfere with plaque removal and can lead to gingival recession and midline diastema. Thus in order to facilitate maintenance of proper oral hygiene and prevention of gingival recession, frenectomy is done.

Aim: The aim of this study was to compare the effects of the diode laser and the scalpel technique on the degree of postoperative pain experienced by patients after frenectomy.

Materials and Methods: Twenty patients requiring frenectomy were randomly assigned to two treatment groups, one with a diode laser and the other with a scalpel. The postoperative pain of each patient was assessed using a Visual Analog Scale and the number of analgesic used was recorded on postoperative days one and seven.

Results: Patients treated with the diode laser had significantly less postoperative pain at day one and used less number of analgesic (2.30 $\pm 0.94)$ as compared to scalpel surgery $(4.40 \pm 1.07)$.

Conclusion: This comparative study indicates that diode laser treatment for frenectomy provides better patient perception in terms of pain compared to scalpel technique.

Keywords: Diode laser; frenectomy; pain perception.
\end{abstract}

\section{INTRODUCTION}

A frenum is a fold of mucous membrane enclosing the muscle fiber that attaches the lips and cheeks to the alveolar mucosa and/or gingiva and underlying periosteum. ${ }^{1}$ Its primary function is to provide stability of the upper and lower lip and the tongue. The frenal attachments have been classified by Placek et $\mathrm{al}^{2}$ as:

1. Mucosal - when the frenal fibres are attached up to the mucogingival junction.

2. Gingival - when the fibres are inserted within the attached gingiva.

3. Papillary - when the fibres are extending into the interdental papilla.

4. Papilla penetrating - when the fibres cross the alveolar process and extend up to the palatine papilla.

Papillary and papilla penetrating frena are considered as pathological as they have been found to be associated with

\section{Correspondence:}

Dr. Soni Bista

Department of Periodontology and Oral Implantology, Universal College of Medical Sciences, College of Dental Surgery, Bhairahawa, Nepal.

email: sonibista12345@gmail.com

\section{Citation}

Bista S, Adhikari K, Saimbi CS, Agrahari B. Comparison of Patient Perceptions with Diode Laser and Scalpel Technique for Frenectomy. J Nepal Soc Perio Oral Implantol. 2018;2(1):6-8. loss of papilla, recession, diastema, difficulty in brushing, malalignment of teeth, compromising the denture fit or retention leading to psychological disturbances to the individual. ${ }^{3}$ In order to facilitate maintenance of proper oral hygiene and to prevent gingival recession, frenectomy can be carried out either by routine scalpel technique, electrosurgery, radiosurgery or by using lasers. ${ }^{4}$

The aim of this study was to compare the effects of the diode laser and the scalpel technique on the patient perceptions of pain after frenectomy procedure.

\section{MATERIALS AND METHODS}

The samples were selected from the patients visiting the Out Patient Department of Periodontology and Oral Implantology, Universal college of Dental Surgery, Bhairahawa, Nepal from November 2017 to January 2018. Twenty systemically healthy patients with good compliance who exhibited papillary and papilla penetrating labial frenal attachments requiring frenectomy were randomly assigned to two groups.

Group A: Comprised of 10 patients selected for diode laser frenectomy (Figure 1).

Group B: Comprised of 10 patients selected for scalpel frenectomy (Figure 2).

Patients who were on any kind of medication were excluded from the study. 


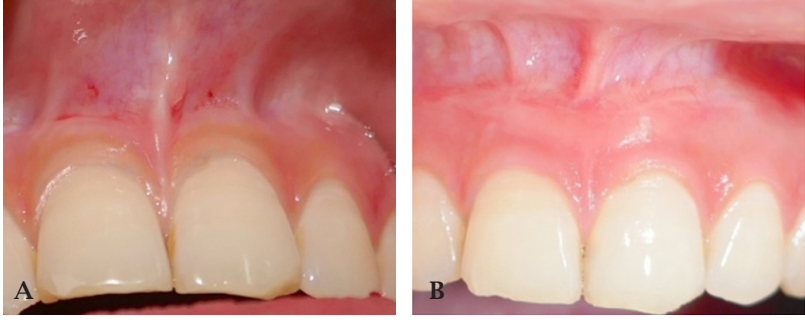

Figure 1 (A): Preoperative view showing papillary frenal attachment. (B) Postoperative view after a month

The study protocol was reviewed and approved by the Institutional Review Committee (IRC) of Universal College of Medical Sciences, Bhairahawa, Nepal. A written informed consent was obtained from all the patients willing to participate in the study. Phase I therapy was carried out for all the patients and were recalled and evaluated for surgical procedure after a month. A complete blood picture was advised for all the patients prior to the surgery.

Diode laser (iLase ${ }^{\mathrm{TM}}$ ) emitting $940 \mathrm{~nm}$ was used in Group A, where preset value was adjusted: Power of 2.00 watt, pulsed contact mode, continuous pulse duration, and pulse interval of $1.00 \mathrm{~ms}$. Safety measures were taken for operator, patient and assistant by wearing the recommended laser protective eyewear. High speed suction and clinical masks was used to prevent infection from the laser plume. For frenectomy, local anesthetic infiltration using 2\% Lignocaine with adrenaline 1:200,000 was administered adjacent to the frenum attachment. Blunt end of the probe was used to check for the objective symptoms. After the area was anesthetized, the incision was carried out using bendable laser tip with diameter of $300 \mu \mathrm{m}$. The intervening labial frenum was released from its apex to the base in a brushing stroke. After excision, the surgical site was wiped off with cotton pellet soaked in normal saline. The entire procedure was painless with no bleeding and lesser intra-operative time. Thus, there was no need for sutures. In Group B, conventional classical technique ${ }^{5}$ was used for frenectomy. Under local infiltration, the aberrant frenum was held with a pair of hemostats, and the whole band oftissue together with its alveolar attachment was excised with a No.15 blade. Periosteal scoring was done to remove any remaining

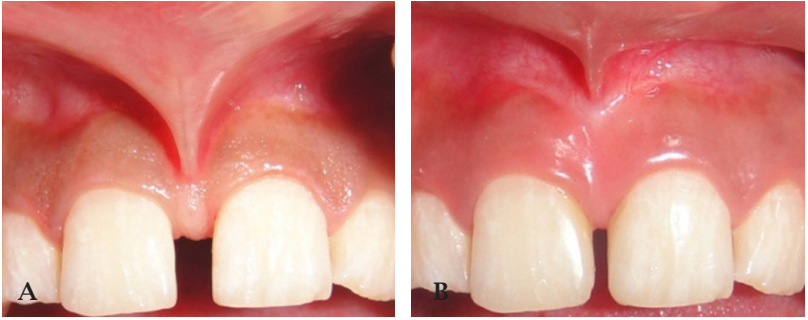

Figure 2(A): Preoperative view showing papilla penetrating type of frenal attachment. (B) Postoperative view after a month

fibrous adhesions to the underlying periosteum. After the bleeding was controlled, the wound was closed with 4-0 silk sutures and dressing (COE-PAK ${ }^{\mathrm{TM}}$ GC America) was given. Both groups received postoperative instructions and analgesics were prescribed to be taken as needed.

At 1st and 7th day postoperatively, patients were recalled and assessed for pain perception. The patients were asked to mark their pain perception on a $10 \mathrm{~cm}$ long Visual Analog Scale (VAS) having two endpoints ranging from 0 to 10 where 0 designates no pain and 10 designates unbearable pain. Similarly, the number of analgesic consumed by each patient was recorded. After completion, all recordings were analyzed, which included comparison of postoperative pain and their number of analgesics taken after the procedure in both the groups.

For statistical analysis, non-parametric tests were chosen for continuous variables because the data were not distributed normally. Comparisons between groups were applied using independent paired t-test. Results were represented as Mean \pm SD and $p$ value $<0.05$ was considered to be significant. Statistical analyses were performed using a Statistical Package for Social Sciences (SPSS) software version 22 (IBM Corporation, Armonk, New York, USA).

\section{RESULTS}

Results of the study are summarized in Table 1 which show mean scores of pain perception after both the techniques. The mean scores of VAS for pain on day one was significantly lower $(\mathrm{p}<0.05)$ in Group A $(3.20 \pm 0.91)$ compared to the Group B $(5.00 \pm 1.49)$. In addition, mean score of number of analgesic used was less in Group A (2.30

Table 1: Mean scores of pain perception after conventional and laser technique.

\begin{tabular}{|c|c|c|c|c|c|}
\hline & Group & $\mathbf{N}$ & Mean \pm SD & Standard Error Mean & Statistical Significance \\
\hline \multirow{2}{*}{ VAS Day 1} & A-LASER & 10 & $3.20 \pm 0.91$ & 0.29059 & \multirow{2}{*}{$\mathrm{p}=0.004 *$} \\
\hline & B-Scalpel & 10 & $5.00 \pm 1.49$ & 0.47140 & \\
\hline \multirow{2}{*}{ VAS Day 7} & A-LASER & 10 & $0.40 \pm 0.51$ & 0.16330 & \multirow{2}{*}{$\mathrm{p}=0.081$} \\
\hline & B-Scalpel & 10 & $1.20 \pm 0.42$ & 0.13333 & \\
\hline \multirow[t]{2}{*}{ Number of analgesic used } & A-LASER & 10 & $2.30 \pm 0.94$ & 0.30000 & $\mathrm{p}=0.000^{*}$ \\
\hline & B-Scalpel & 10 & $4.40 \pm 1.07$ & 0.33993 & \\
\hline
\end{tabular}

$* p \leq 0.05$ 
$\pm 0.94)$ compared to Group B $(4.40 \pm 1.07)$ which was highly significant $(\mathrm{p}<0.00)$. Although mean score of Visual Analog Scale (VAS) of pain on day seven was less in Group A (0.40 $\pm 0.51)$ than in Group B $(1.20 \pm 0.42)$, the difference was not statistically significant $(\mathrm{p}=0.081)$.

\section{DISCUSSION}

In the present study, patients treated with the diode laser had significantly less $(\mathrm{p}<0.05)$ postoperative pain at day one as compared to scalpel surgery which was consistent with the result of the study done by Haytac et al. ${ }^{6}$ The decreased pain perception seen after using laser in the present study may be due to the reason given by Fisher et $\mathrm{al}^{7}$ as protein coagulum is formed on the wound surface which acts as a biologic dressing and seals the ends of the sensory nerves. On the contrary, the increased pain perception with the scalpel frenectomy appreciated in our study might be attributed to the fact that was given by Buchibabu et $\mathrm{al}^{8}$ stating it as a more intrusive surgical procedure involving blood loss with wide surgical wound and suturing.

However, there was no statistical significant difference in postoperative pain on 7th day, supported by the study done by Kaur et al. ${ }^{9}$ Although, contrary evidence also exists. ${ }^{6}$

This study also revealed that patients from the laser group used less number of analgesics than scalpel group which was highly significant which was observed in the study done by Patel et al. ${ }^{4}$ But no significant difference was observed by others ${ }^{9}$ when using analgesic by patients treated with laser compared to scalpel frenectomy.

The laser technique offers some advantages such as it causes relatively bloodless surgical and postsurgical event, has the ability to precisely coagulate, vaporize, or cut tissue, there is minimal swelling, scarring, mechanical trauma without suturing in most cases and reduction of surgical time, postsurgical pain with high patient acceptance. ${ }^{10}$ In the present study, patients treated with diode laser had less bleeding and it was easier to perform with less intraoperative time than conventional scalpel surgery.

\section{CONCLUSION}

This clinical study indicates that diode laser treatment for frenectomy provides better patient perception in terms of pain compared to scalpel technique.The inclusion of microbiological parameter would have supported the clinical findings to assess healing after the frenectomy procedure by both the techniques. Hence,further longitudinal studies with larger sample size are needed to establish the exact efficacy of diode laser technique over conventional scalpel technique for frenectomy procedure.

\section{REFERENCES}

1. Abullais SS, Dani N, Ningappa P, Golvankar K, Chavan A, Malgaonkar N, et al. Paralleling technique for frenectomy and oral hygiene evaluation after frenectomy. J Indian Soc of Periodontol. 2006; 20(1):28-31.

2. Mirko P, Miroslav S, Lubor M. Significance of the labial frenum attachment in periodontal disease in man. Part 1. Classification and epidemiology of the labial frenum attachment. J Periodontol. 1974;45(12):891-4.

3. Patel RM, Varma S, Suragimath G, Abbayya K, Zope SA, Kale V. Comparison of labial frenectomy procedure with conventional surgical technique and diode laser. J Dent Lasers. 2015;9(2):94.

4. Gandhi D, Gandhi P. Comparision of Healing Period after Frenectomy Using Scalpel, Electrocautery \& Diode Laser. Br J Med Med Res. 2017;21(12):1-9.

5. Archer WH. Oral surgery for a dental prosthesis. In: Archer WH (ed). Oral and Maxillofacial surgery.5th ed. Philadelphia: WB Saunders Co; 1975.p.135-210.

6. Haytac MC, Ozcelik O. Evaluation of patient perceptions after frenectomy operations: A comparison of carbon dioxide laser and scalpel techniques. J Periodontol. 2006;77:1815-9.

7. Fisher SE, Frame JW, Browne RM, Tranter RM. A comparative histological study of wound healing following CO2 laser and conventional surgical excision of canine buccal mucosa. Arch Oral Biol. 1983;28:287-91.

8. Butchibabu K, Koppolu P, Mishra A, Pandey R, Swapna LA, Uppada UK. Evaluation of patient perceptions after labial frenectomy procedure: A comparison of diode laser and scalpel techniques. Eur J Gen Dent. 2014;3:129-33.

9. Kaur P, Dev YP, Kaushal S, Bhatia A, Vaid R, Sharma R. Management of the upper labial frenum: a comparison of conventional surgical and lasers on the basis of visual analogue scale on patients perception. J Periodontal Med Clin Pract. 2014;1(1):38-44.

10. Goharkhay K, Moritz A, Wilder Smith P, Schoop U, Kluger W, Jakolitsch S, et al. Effects on oral soft tissue produced by a diode laser in vitro. Lasers Surg Med. 1999;25:401-6. 